\title{
Analysis of Singularities for Partial Differential Equations
}




\section{Series in Applied and Computational Mathematics}

ISSN: 2010-2739

Series Editors: Philippe G. LeFloch (University of Paris 6, France)

Jian-Guo Liu (Duke University, USA)

Vol. 1: Analysis of Singularities for Partial Differential Equations

by Shuxing Chen (Fudan University, China) 
Analysis of

Singularities for Partial Differential Equations

\section{Shuxing Chen}

Fudan University, China 
Published by

World Scientific Publishing Co. Pte. Ltd.

5 Toh Tuck Link, Singapore 596224

USA office: 27 Warren Street, Suite 401-402, Hackensack, NJ 07601

UK office: 57 Shelton Street, Covent Garden, London WC2H 9HE

\title{
British Library Cataloguing-in-Publication Data
}

A catalogue record for this book is available from the British Library.

\begin{abstract}
ANALYSIS OF SINGULARITIES FOR PARTIAL DIFFERENTIAL EQUATIONS Series in Applied and Computational Mathematics — Vol. 1

Copyright (C) 2011 by World Scientific Publishing Co. Pte. Ltd.

All rights reserved. This book, or parts thereof, may not be reproduced in any form or by any means, electronic or mechanical, including photocopying, recording or any information storage and retrieval system now known or to be invented, without written permission from the Publisher.
\end{abstract}

For photocopying of material in this volume, please pay a copying fee through the Copyright Clearance Center, Inc., 222 Rosewood Drive, Danvers, MA 01923, USA. In this case permission to photocopy is not required from the publisher.

ISBN-13 978-981-4304-83-2

ISBN-10 981-4304-83-2

Printed in Singapore. 


\section{Preface}

Regularity or singularity is a most important property of solutions to partial differential equations. In the study of the theory of partial differential equations and its applications people often confront various problems, which require to look for solutions. Besides the existence and uniqueness of solutions to these problems it is also essentially significant to master or understand various properties of solutions including regularity and singularity, periodicity, asymptotic behavior etc. Among these properties the regularity or singularity of the solutions often plays a crucial role or occupies in the core position due to the following reasons:

1. In the study of the modern theory of partial differential equations the concept of distribution is extensively applied. Correspondingly, the proof of the existence of solutions to various problems is often decomposed to two steps. The first step is to prove the existence of a distribution solution in a very weak sense, then the second step is to improve the regularity of the given solution, so that the weak solution will also be a solution in strong sense or even in classical sense. Obviously, the second step is nothing but the study of regularity of solutions.

2. Regularity and singularity are merely two sides of one matters. In many applications more attentions are often paid to the formation and the distribution of singularities of solutions. For instance, in the material sciences the singularity often corresponds to the crack of materials, in chemical reaction the formation of singularity often corresponds to explosion, and in gas dynamics the formation of singularity often corresponds to the formation of shock. In the problems on wave propagation the singularity of solutions can describe the wave front, so that the propagation of singularities can describe the physical phenomena of wave propagation precisely. 
3. The regularity of a solution gives us the information whether the solution is continuous or smooth (having continuous derivatives). If the solution is continuous, then one can use the value of the solution at a given point to stand for its value in a neighborhood of this point. This gives us great convenience to understand the solution quantitatively, as well as to compute the solution numerically.

Further study also finds that the regularity of solutions is closely related to the existence and uniqueness of solutions. When various methods of functional analysis are applied in the study of partial differential equations one usually has to fix a functional space, to which the expected solution belongs. Such a space automatically implies a kind of regularity of the solution. Besides, the regularity of solutions is often expressed by some estimates, which are also helpful in the proof of existence and uniqueness of solutions.

Many textbooks and monographs on partial differential equations contain some discussions on regularity and singularity of solutions, but so far there is not any book especially expounding such a topic. Our book is named as Analysis of singularities for partial differential equations, because the crucial points, as well as the main difficulties, in the study of partial differential equations, are often companied by the formation, development and propagation of singularities. In this book we are trying to emphasize the importance of the study of this topic with offering a systematical results and methods on some typical problems. However, due to the rapid development of the study in this area, we can only give a general survey on the study of other problems. We hope both the detailed analysis on typical problems and the survey on other problems are helpful for reader's further research.

The writing of the book is partially supported by National Natural Science Foundation of China, the Key Grant of National Basic Research Program of China and the Doctoral Foundation of National Educational Ministry. 


\section{Contents}

Preface $\quad$ v

1. Introduction to problems on singularity analysis 1

1.1 The classical singularity propagation theorem . . . . . . . . 1

1.2 Towards to modern theory . . . . . . . . . . . . 9

2. Singularity analysis for linear equations $\quad 13$

2.1 Wave front set . . . . . . . . . . . . . . 13

2.2 Singularity propagation theorem for equations of principal

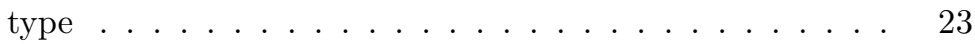

2.3 Reflection of singularity on boundary . . . . . . . . 30

2.4 Further discussions . . . . . . . . . . . . . . . . 43

2.4.1 Generalized reflection of singularity on boundary . 43

2.4.2 The operators with multiple characteristics . . . 46

3. Singularity analysis for semilinear equations 49

3.1 Theorem of propagation of 2 s weak singularity . . . . . 50

3.2 Theorem on propagation of 3 s weak singularity . . . . . 57

3.3 Singularity interaction and singularity index . . . . . . . 62

3.4 Propagation of conormal singularity . . . . . . . . . 73

3.5 Interaction of conormal singularities $\ldots \ldots \ldots \ldots$

3.5.1 Extension of the concept of conormal singularities 80

3.5.2 Pseudo-composition . . . . . . . . . . 86

3.5.3 Theorem on interaction of conormal singularities . 87

3.5.4 Reflection of conormal singularities . . . . . . . 90 
4. Propagation of singularities for fully nonlinear equations

4.1 Theorem of propagation of singularities for principal type

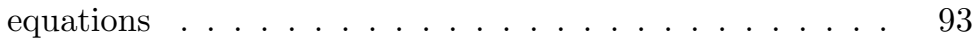

4.2 Propagation of conormal singularities for nonlinear equations . . . . . . . . . . . . . . . 101

5. Propagation of strong singularities for nonlinear equations

5.1 Solutions with fan-shaped singularity structure of semilinear equations . . . . . . . . . . . . . . . 112

5.2 Solutions with flower-shaped singularity structure of semilinear equations . . . . . . . . . . . . . . . 122

5.3 Solutions with strong singularities of quasilinear equations (1-d case) . . . . . . . . . . . . . . . . 131

5.4 Solutions with strong singulari ties of quasilinear equations (m-d case) . . . . . . . . . . . . . . . 137

5.4.1 Fan-shaped singularity structure . . . . . . . . 137

5.4.2 Flower-shaped singularity structure . . . . . . . . 142

6. Formation of shocks for quasilinear hyperbolic equations 147

6.1 The case of scalar equation _ . . . . . . . . . . . 147

6.1.1 Two mechanism of blow-up of smooth solutions . 147

6.1.2 Formation of a shock . . . . . . . . . . . . . 149

6.1.3 Estimates of the solution in the neighborhood of the starting point of shock . . . . . . . . 156

6.2 The case of system . . . . . . . . . . . . . . . 159

6.2.1 Background and conclusion . . . . . . . . . 160

6.2.2 The property of the first approximate solution . . 163

6.2.3 Estimates and convergence of the sequence of approximate solutions . . . . . . . . . . . . . 169

6.2.4 The case for full Euler system . . . . . . . . . 177

$\begin{array}{lll}\text { Appendix A } & \text { Brief review on paradifferential operators } & 181\end{array}$

A.1 Diadic decomposition . . . . . . . . . . . . . 181

A.2 Paradifferential operators and paralinearzation . . . . . . 185

A.3 Paracomposition . . . . . . . . . . . . . . . 189 